\title{
Going both ways: immune regulation via CD1d-dependent NKT cells
}

\author{
Dale I. Godfrey ${ }^{1}$ and Mitchell Kronenberg ${ }^{2}$
}

1Department of Microbiology and Immunology, University of Melbourne, Parkville, Victoria, Australia. ${ }^{2}$ La Jolla Institute for Allergy and Immunology, San Diego, California, USA.

\begin{abstract}
NKT cells are a unique $T$ lymphocyte sublineage that has been implicated in the regulation of immune responses associated with a broad range of diseases, including autoimmunity, infectious diseases, and cancer. In stark contrast to both conventional $\mathrm{T}$ lymphocytes and other types of Tregs, NKT cells are reactive to the nonclassical class I antigen-presenting molecule CD1d, and they recognize glycolipid antigens rather than peptides. Moreover, they can either up- or downregulate immune responses by promoting the secretion of Th1, Th2, or immune regulatory cytokines. This review will explore the diverse influences of these cells in various disease models, their ability to suppress or enhance immunity, and the potential for manipulating these cells as a novel form of immunotherapy.
\end{abstract}

\section{Introduction}

NK T cells (NKT cells) are, by definition, T lymphocytes, as they express a TCR. This distinguishes them from NK cells, although NKT cells share some markers characteristic of NK cells such as CD161 (CD161c is known as NK1.1 in mice) or NKR-P1 (reviewed in ref. 1). In contrast to conventional $\mathrm{T}$ lymphocytes and other Tregs, the NKT cell TCR does not interact with peptide antigen presented by classical MHC-encoded class I or II molecules, but instead it recognizes glycolipids presented by CD1d, a nonclassical antigen-presenting molecule (reviewed in refs. 2 and 3). NKT cells also express a far more limited range of TCR variable $(V)$ region genes. In mice, most express an invariant TCR $\alpha$ chain rearrangement $(V \alpha 14-J \alpha 18)$ with a conserved CDR3 region, and they typically coexpress $V \beta 8.2, V \beta 2$, or $V \beta 7$. Similar T cells are present in other mammalian species, including humans. The homologous population of human NKT cells express a $V \alpha 24-J \alpha 18$ rearranged TCR $\alpha$ chain with a $V \beta 11$-containing $\beta$ chain $(4,5)$. The evolutionary conservation of these cells is striking, as mouse NKT cells recognize human CD1d plus glycolipid antigen and vice versa (6). This alone is sufficient to suggest that these cells play a critical, and probably unique, role in the immune system.

In reviewing immune regulation by NKT cells, it is first necessary to clarify the definition of the term. It is apparent that the simple classification of NKT cells as T lymphocytes that also express NK receptors is inadequate. We have discussed this issue in greater detail elsewhere (1), but the main problems with this definition are that many conventional $\mathrm{T}$ cells upregulate NK receptors, including NK1.1/CD161, upon activation, while many CD1d-dependent NKT cells do not express them. This article will primarily focus on the group of cells that are CD1d dependent and express an invariant Vo14-J $\alpha 18$ (mice) or Va24J $\alpha 18$ (humans) TCR rearrangement. These are variously known

Nonstandard abbreviations used: ACAID, anterior chamber-associated immune deviation; CD40L, CD40 ligand; $\alpha$-GalCer, $\alpha$-galactosylceramide; EAE, experimental autoimmune encephalomyelitis; GVHD; graft versus host disease; NKT cells, NK T cells; $V$, variable.

Conflict of interest: The authors have declared that no conflict of interest exists.

Citation for this article: J. Clin. Invest. 114:1379-1388 (2004).

doi:10.1172/JCI200423594 as classical NKT cells, V $\alpha 14$ invariant (V $\alpha 14 i)$ NKT cells, invariant NKT cells (iNKT cells), or type I NKT cells (reviewed in refs. 1,3 , and 7 ), but for the purposes of this review, the simple term NKT cells will represent solely these cells. It is reasonable to emphasize this subgroup not only because of its distinct characteristics, but because most of the literature on immune regulation by NK1.1- or CD161-expressing T lymphocytes concerns these cells. Moreover, in C57BL/6 mice that express NK1.1, the majority of NK1.1-expressing T cells are in fact this CD1d-reactive subgroup (8-10). In humans, however, a much larger set of T cells expresses CD161, and the V $\alpha 24 / V \beta 11$-expressing subset is only a minority (11-13).

NKT cells include at least 2 subsets, distinguishable as $\mathrm{CD}^{+}$ or $\mathrm{CD}^{-}$, although in humans and monkeys, but not mice, some of these cells express CD8 (13). Both the $\mathrm{CD}^{+}$and $\mathrm{CD}^{-}$subsets of NKT cells exist wherever other $T$ cells are found, although the relative frequency of these cells varies in a tissue-specific manner $(8,10)$. In mice, NKT cells are found at the highest frequency in liver (10-40\% of liver lymphocytes), but they are present at lower frequencies (typically $<1 \%$ ) in thymus, bone marrow, spleen, lymph node, and blood. The tissue distribution of NKT cells in humans has not been as extensively studied, although they are clearly less frequent $(<1 \%)$ in human liver compared with the same tissue in mice $(11,14,15)$. The significance of species-specific differences in NKT cell frequencies is unknown.

The recognition of glycolipid antigens in association with CD1d means that NKT cells recognize a class of antigens ignored by conventional $\mathrm{T}$ cells. Relatively few examples of these antigens are defined, although presentation of several mycobacterial cell wall antigens by the 3 other human CD1 molecules, CD1a, $\mathrm{CD} 1 \mathrm{~b}$ and $\mathrm{CD} 1 \mathrm{c}$, has been well characterized (reviewed in ref. 16), as has presentation of several autologous antigens, including gangliosides and some phospholipids. The compound most efficient for activating the majority of NKT cells is a synthetic glycolipid (originally derived from a marine sponge) known as $\alpha$-galactosylceramide ( $\alpha$-GalCer) (reviewed in refs. 17 and 18). This compound binds effectively to CD1d, and the complex of glycolipid plus CD1d binds the NKT cell TCR (19), leading to activation of these cells in both mice and humans. In fact, NKT cells in mice and humans can be best identified by binding of 
Table 1

Comparison of NKT cells and CD25+CD4+ Tregs

\begin{tabular}{|c|c|c|}
\hline & NKT cells & CD25+CD4+ Treg \\
\hline \multicolumn{3}{|l|}{ Distribution and specificity } \\
\hline $\begin{array}{l}\text { Prevalence } \\
\text { (spleen and thymus) }\end{array}$ & $0.5-1 \%$ & $5-10 \%$ \\
\hline Specificity & Glycolipids plus CD1d & Peptides plus MHC class II \\
\hline Autoreactivity & Yes & Yes, in some cases \\
\hline TCRs & Invariant $V_{\alpha}$, limited $V_{\beta}$ & Diverse \\
\hline \multicolumn{3}{|l|}{ Development } \\
\hline Positive selection & CD1d-expressing thymocytes & MHC class II+ thymic epithelium \\
\hline Unique requirements & $\begin{array}{l}\text { IL-15 pathway, lymphotoxin, } \\
\text { fyn, Ets, AP-1, and NF- } \mathrm{B} B \\
\text { transcription factors. CD1d antigen } \\
\text { presentation pathway(?): } \\
\text { cathepsin L, AP-3, saposins }\end{array}$ & $\begin{array}{l}\text { IL-2, foxp3, } \\
\text { B7-mediated costimulation }\end{array}$ \\
\hline Ontogeny & First week after birth & First week after birth \\
\hline \multicolumn{3}{|l|}{ Function } \\
\hline Mode of action & Suppression or activation & Suppression \\
\hline Cytokines & Many, including Th1 and Th2 & IL-10, TGF- $\beta$ \\
\hline $\begin{array}{l}\text { Function-related } \\
\text { cell-surface molecules }\end{array}$ & CD40L, FAS-L & TGF- $\beta$, CTLA-4 \\
\hline
\end{tabular}

FAS-L, Fas ligand.

this problem requires careful analysis of NKT cells at the single-cell level (using intracellular cytokine staining or similar approaches) to monitor the changes that occur in individual NKT cells over time and in response to different types of stimuli.

NKT cells influence and regulate a wide range of immune responses. Table 1 shows a comparison between them and $\mathrm{CD} 25^{+} \mathrm{CD} 4^{+}$Tregs, which, like NKT cells, also regulate different types of immune responses and which also have recently attracted much attention. There are some clear similarities between these $2 \mathrm{~T}$ lymphocyte subtypes, including expression of CD25 by human CD $4^{+}$NKT cells (32). We also find in mice that while CD25 is expressed at low levels by NKT cells, it is upregulated upon activation in vivo (D.I. Godfrey and R. Keating, unpublished data). This raises the possibility of confusion between these distinct types, such that some activity ascribed to $\mathrm{CD} 25^{+} \mathrm{CD} 4^{+}$Tregs might really be due to NKT cells. It is therefore important to carefully dis-

fluorochrome-conjugated, tetrameric complexes of CD1d loaded with $\alpha$-GalCer $(9,20,21)$. $\alpha$-GalCer has been used in many different studies and is a very potent NKT cell agonist (17). Many other glycolipid molecules have been tested for NKT cell stimulatory capacity, with some, including ganglioside GD3 (22), glycophosphatidylinositol $(23,24)$, phosphoethanolamine $(25)$, and some forms of $\beta$-GalCer (26), activating subsets of these cells (22-26).

When activated, NKT cells respond with vigorous cytokine production within 1-2 hours of TCR ligation (reviewed in refs. 2 and 3 ). These cells release Th1-type cytokines including IFN- $\gamma$ and TNF, as well as Th2-type cytokines including IL-4 and IL-13 (reviewed in refs. 27 and 28), and at least in mice, they seem to store or pre-form some mRNA for these cytokines even before activation with exogenous antigens $(29,30)$. Moreover, individual NKT cells are able to make both Th1- and Th2-type cytokines simultaneously following stimulation in vivo $(9,29,31)$, an unusual characteristic that at face value seems paradoxical, as Th1 cytokines often antagonize the action of Th 2 cytokines and vice versa. Despite this Th0-like cytokine pattern, NKT cells can "go both ways," as their activation in some cases can polarize the immune response in a Th1 direction, while in other cases a Th2 response is generated. Adding to the unpredictability surrounding the functional consequences of NKT cell activation, these lymphocytes have been implicated as immunosuppressive cells in some systems, usually via their production of the Th2-type cytokines or IL-10, while in other systems, they appear to promote enhanced cell-mediated immunity via production of Th1type cytokines (reviewed in refs. 27 and 28). The mechanisms that determine the cytokine polarity of the NKT cell response, and the influence of the NKT cells response on the systemic immune system, are not well understood, and indeed, this problem represents a key challenge in the field of NKT cell research. Resolving tinguish these cells, and the table highlights their similarities and, more importantly, their differences.

\section{NKT cell-mediated suppression of tissue destruction}

The potent production of immunosuppressive cytokines such as IL-4, IL-10, and IL-13 by NKT cells prompted speculation that these cells might be able to suppress cell-mediated immune responses. Over the last few years, this has been demonstrated in several different models.

The introduction of antigen into the anterior chamber of the eye in mice results in local and systemic inhibition of antigenspecific inflammatory $T$ cell responses, known as anterior chamber-associated immune deviation (ACAID). This is thought to be one of several mechanisms responsible for the immune-privileged status of this organ. A series of studies in which the nominal antigen OVA is injected into the anterior chamber of WT and NKT cell-deficient (CD1 $\left.d^{--}\right)$mice has revealed that ACAID is an NKT cell-dependent phenomenon. There is evidence that this may normally be a thymus-dependent step in which tolerogenic APCs traffic from the eye to the thymus, which leads to thymic NKT cell migration to the periphery $(33,34)$. However, adoptive transfer of NKT cells directly from spleen of naive mice to NKT cell-deficient mice can restore ACAID (35), and NKT cell-derived IL-10 was critical for this effect (36). The antigen specificity of the suppression observed in this model is mediated by $\mathrm{CD}^{+}$Tregs that seem to be induced by NKT cells (37), possibly as a direct result of exposure to NKT cell-derived IL-10. This would explain how NKT cells, which themselves are unlikely to be protein antigen specific, can promote protein antigen-specific suppression. Interestingly, this also suggests that different Treg populations might sometimes work as part of an integrated system. Other examples of this phenomenon 
exist, such as suppression of NKT cell activity by $\mathrm{CD} 25^{+}$Tregs in mice and humans $(38,39)$.

Data from experimental mouse models suggest that NKT cells may mediate allograft tolerance. Corneal allografts represent one of the most readily accepted types of solid tissue grafts. In a mouse corneal allograft model, BALB/c mouse corneas were grafted to WT or TCR $J \alpha 18^{-/-}$C57BL/ 6 recipients, which do not have NKT cells because they cannot form the invariant $V \alpha 14-J \alpha 18$ rearrangement. In this system, NKT cells were required for allograft tolerance (40) and, as is the case with ACAID, this involved NKT celldependent generation of downstream allospecific Tregs.

NKT cells have been shown to participate in 2 different models of cardiac allograft tolerance following tolerogenic immunotherapy. In both models, the suppressive influence of these cells was only observed in association with immunological conditioning regimens, which were effective in the presence but not the absence of NKT cells. One study (41) showed that immunological blockade of lymphocyte function adhesion molecule-1/ICAM-1 (LFA-1/ICAM-1) or CD28/B7 interactions could inhibit cardiac allograft rejection in WT mice but not in $J \alpha 18^{-/-}$mice. Another study showed that a conditioning regimen involving fractionated lymphoid irradiation, partial $\mathrm{T}$ cell depletion, and infusion of donor bone marrow cells successfully permitted cardiac allograft tolerance in mice, but, again, this required the presence of NKT cells, as this regimen did not work in $C D 1 d^{-/-}$or $J \alpha 18^{-/-}$mice (42). A similar tolerogenic influence of NKT cells was reported in a model of xenograft tolerance, where an anti-CD4 antibody-mediated tolerogenic regime was able to promote survival of rat xenogeneic pancreatic islet grafts in WT but not J $\alpha 18^{-/-}$mice (43).

Bone marrow-derived NKT cells have been shown to potently inhibit graft versus host disease (GVHD) in a mouse model where NKT cells were cotransferred with allogeneic $(\mathrm{C} 57 \mathrm{BL} / 6)$ bone marrow into lethally irradiated recipient (BALB/c) mice (44). Similar results were reported by the same group using a different model of GVHD suppression, involving nonmyeloblative, fractionated lymphoid irradiation and antibody-mediated $\mathrm{T}$ cell depletion (45). In both studies, inhibition was critically dependent on the ability of the NKT cells to produce IL-4 $(44,45)$. Moreover, if these cells were isolated from $I L-4^{-/-}$mice, GVHD was exacerbated rather than diminished, presumably due to their intact production of Th1 cytokines such as IFN- $\gamma(44)$. The IL-4 dependence not only indicates that other NKT cellderived immunosuppressive cytokines, such as IL-10 and IL-13, were insufficient for suppression of GVHD in these models, it also suggests that IL-4 production by NKT cells dominates the response, counteracting the potential Th1-like influence by these cells, at least in this model.

In most of the above studies where NKT cells were responsible for the promotion and/or maintenance of immunological tolerance, they did this in the absence of intentional exogenous stimulation. Indeed, $\alpha$-GalCer treatment was unable to improve cardiac allograft survival in mice (41). Furthermore, in a study of maternal-fetal tolerance, although NKT cells did not appear to play a decisive physiological role, treatment of the pregnant mice with $\alpha$-GalCer almost invariably triggered abortion (46), which indicates that intentional stimulation of NKT cells by this glycolipid that strongly activates NKT cells does not necessarily promote a tolerogenic effect, although a weaker stimulation by natural or endogenous glycolipids might do so.
While the above studies are all rodent based, and there are currently no comparable studies in human tissue graft recipients, the clear functional overlap between NKT cells in humans and mice makes this an important area of investigation with obvious clinical potential.

\section{NKT cells and autoimmunity}

There is evidence from some autoimmune disease models suggesting that NKT cells naturally influence autoimmunity, while in other models, intentional activation of NKT cells by $\alpha$-GalCer is required to elicit their regulatory function. Until we understand the factors that naturally lead to NKT cell activation, the basis for their involvement in some but not all autoimmune diseases will remain elusive. Nonetheless, as a further illustration of the "going both ways" phenomenon, in some cases NKT cells promote autoimmunity, even in the absence of $\alpha$-GalCer stimulation. This highlights the unpredictable nature of the NKT cell influence on immune responses and the importance of being able to understand the basis for the regulation of their cytokine production as well as to harness their activity and drive them in the desired direction.

The greatest number of studies investigating the role of NKT cells in autoimmune disease pathogenesis has involved type 1 diabetes. NKT cells are reduced in diabetes-prone NOD mice (reviewed in refs. 26, 47, and 48), and increasing the number of NKT cells by adoptive transfer (49-51) or via the introduction of a V $\alpha 14-J \alpha 18$ transgene (51) reduced disease progression. Additionally, NKT cells from NOD mice produce less IL-4 after stimulation $(20,50,52-56)$. Nonetheless, stimulation of these cells via repeated administration of $\alpha$-GalCer has a therapeutic effect $(53,55-57)$. In a number of studies, protection from diabetes by NKT cells was associated with the induction of a Th2 response to islet autoantigens $(53,56)$. In a transfer model, however, activation of diabetogenic $\mathrm{T}$ cells given to NOD mice in the presence of NKT cells inhibited differentiation of the former into IFN- $\gamma$ producers by preventing their expansion and proliferation, but the NKT cells did not act through IL-4 production (58). NKT cell activation leads to changes in other populations, including NK cells and DCs. In another study, treatment with $\alpha$-GalCer led to increased numbers of CD8 $\alpha^{-}$myeloid DCs in the pancreatic lymph nodes, and transfer of myeloid DCs from the pancreatic lymph node of untreated mice prevented diabetes in recipients (57). Albeit indirectly, these data implicate a role for regulatory DCs acting downstream of NKT cell activation. Thus, in addition to Th2 deviation, NKT cells may act by inducing anergy in isletspecific T cells or by altering the function of APCs.

The clinical relevance of NOD mouse studies was supported when the frequency of invariant V $\alpha 24-J \alpha 18$ sequences among $\mathrm{V} \alpha 24^{+}$-double-negative (DN) T cells was reported to be lower in diabetics than their disease-free identical twins or triplets (59). The ability of NKT cell clones derived from diabetics to produce IL-4 also was impaired. These findings were supported by 1 study (60), but contradicted by 2 others $(61,62)$. The discrepancy could be due to differences in the patient and control populations, different methods of detection of NKT cells in the different human studies, and problems related to the comparison of analyses of peripheral blood of humans to analyses of lymphoid tissues in mice. The latter concern is highlighted by the fact that while NOD mice are deficient for NKT cells in thymus, liver, and spleen, NKT cells in peripheral blood are at normal or even higher levels than in other commonly used mouse strains (63). 
Experimental autoimmune encephalomyelitis (EAE) is an animal model of MS caused by immunizing susceptible rodent strains with myelin-derived antigens. The results obtained from NKT cell stimulation by $\alpha$-GalCer treatment in EAE have also been conflicting. $\alpha$-GalCer was found to prevent disease (64-66), to have no effect $(67,68)$, or, in one protocol, to accelerate disease (65). This could be due to differences in the models used or the timing (65) or dose and route (64) of $\alpha$-GalCer. Similar to the findings in NOD mice, $\alpha$-GalCer usually was effective in preventing EAE by shifting the balance from a pathogenic Th1 toward a Th2 response to CNS antigens $(65,66)$. An analog of $\alpha$-GalCer known as OCH seems to preferentially promote IL-4 production both in vitro and in vivo and was found to be more effective than $\alpha$-GalCer in preventing EAE (68) and collagen-induced arthritis (69). It is not yet clear whether OCH alters NKT cell cytokine production directly or whether it acts by affecting the cytokine production of cells activated by NKT cells. Although serum IL-4 and IFN- $\gamma$ levels, within 90 minutes of $\mathrm{OCH}$ administration, were not greatly different from those induced by $\alpha$-GalCer (70), IFN- $\gamma$ levels continue to increase until 12-24 hours following $\alpha$-GalCer but not $\mathrm{OCH}$ treatment (70). Whether this longer-term effect solely reflects an altered NKT cell response or, alternatively, reduced IFN- $\gamma$ production by bystander cells, known to occur following $\alpha$-GalCer administration $(71,72)$, remains to be determined. The influence of IL- 4 and IFN- $\gamma$ production by NKT cells in EAE is not always as expected. One study suggested that NKT cell-derived IFN- $\gamma$ exacerbated EAE, while NKT cell-derived IL-4 suppressed this disease (67); another showed that anti-IL-4 treatment had no effect on $\alpha$-GalCer-mediated inhibition of EAE, while antiIFN- $\gamma$ treatment blocked this effect (64). Furthermore, in V $\alpha 14$ Ja18 transgenic mice, where enhanced NKT cell numbers led to impaired EAE induction without exogenous NKT cell stimulation, protection was associated with a reduction in IFN- $\gamma$ production by autoantigen-specific $T$ cells rather than a Th2 bias in their response, and NKT cell IL-4 was not required (73). A decrease in Vo24-J 18 mRNA in the peripheral blood of MS patients has been found, and short-term lines of NKT cells from MS patients in remission had a Th2 cytokine bias compared with similar lines from relapsed patients, which suggests a regulatory role for these cells in this human disease (74).

An influence of NKT cells in prevention of lupus was suggested in 2 early reports using mouse models of lupus $(75,76)$, although NKT cells were not well defined in these studies. More recently, CD1d deficiency has been associated with exacerbated lupus in a hydrocarbon oil-induced model (77) and more severe skin disease in the lupus-prone MRL $l p r / l p r$ mice (78), although the latter results are at odds with an earlier study that failed to show an effect due to CD1d deficiency in MRL $l p r / l p r$ mice (79). The reason for these inconsistencies is unclear but may be due to differential contamination of unrelated susceptibility/ resistance genes onto the MRL $l p r$ strain background during the backcrossing process to generate CD1d-deficient MRL $l p r / l p r$ mice. Stimulation of NKT cells with $\alpha$-GalCer ameliorated dermatitis in MRL $l p r / l p r$ mice yet surprisingly had no effect on lupus nephritis in the same mice (80). Compared with these disease-preventing influences, NKT cells have the opposite effect, as they have been proposed to promote autoimmunity in (NZB $\times$ NZW) $F_{1}$ mice (81). NKT cells increased in number after the onset of disease, and their transfer from diseased mice to young recipients induced proteinuria and swelling of glomeruli. Fur- thermore, in vivo treatment with an anti-CD1d antibody had beneficial effects in this model (82). Thus, the model seems to create an environment that favors the immune potentiating function, rather than suppressive function, of NKT cells, due to a mechanism that may involve IFN- $\gamma$ secretion and the promotion of the synthesis of pathogenic IgG2a isotype anti-DNA Igs. In colitis induced by dextran sodium sulfate, activation of NKT cells was partially protective (83). Interestingly, NKT cells were required, even in the absence of $\alpha$-GalCer activation, for colitis induced by the hapten oxazalone (84), and NKT cell-derived IL-13 was considered to be critical in this model.

Human NKT cells are reduced in the peripheral blood of patients with a variety of organ-specific and systemic autoimmune conditions but not in association with Graves disease, myasthenia gravis, and celiac disease (85). Limitations of these studies, however, include the very low frequency and variability in the number of NKT cells in normal humans, assessment of the number of cells rather than their function in some cases, and the possibility that peripheral blood might not be representative of the site of disease. For example, in primary biliary cirrhosis, NKT cells were increased in patients in the liver, the target organ, but not in the peripheral blood (86). Again, it is also relevant to point out that NKT cells are reduced in thymus, spleen, and liver of NOD mice but are present at similar levels to other strains in peripheral blood (63).

Clearly there is ample evidence that NKT cells may influence the outcome of autoimmunity, but this may be to the benefit or detriment of the host. It is therefore important to develop a stronger understanding of the mechanisms leading to NKT cell activation in association with autoimmune disease, as well as the physiological factors that drive these cells to suppress or enhance the autoimmune response. Further studies into the behavior of these cells, and the influence of different exogenous stimuli, in the different mouse models of autoimmune disease are required before NKT cells can be safely targeted as an approach to autoimmune disease therapy in the clinic.

\section{NKT cells and the antitumor response}

The potential importance of NKT cells in tumor rejection has been established (87). NKT cells were found to be necessary for IL-12-mediated tumor therapy in mice (88), and the earliest and best-documented effect of $\alpha$-GalCer as an immunotherapeutic agent is its ability to promote NKT cell-dependent rejection of a broad range of experimental tumor lines, including melanoma, thymoma, carcinoma, and sarcoma $(17,87,89)$. Recent evidence has also shown that $\alpha$-GalCer treatment can protect against spontaneous, carcinogen-, or oncogene-induced primary tumor formation in mice (90). Where the mechanisms have been studied in detail, it appears that NKT cell-derived IFN- $\gamma$ production is critical for $\alpha$-GalCer therapy, while other potentially tumoricidal products of NKT cells, such as TNF, Fas ligand, and perforin, seem dispensable $(71,91)$. Consistent with a prime role for IFN- $\gamma$ in NKT cell-mediated tumor responses, a C-glycoside analog of $\alpha$-GalCer that preferentially stimulated IFN- $\gamma$ production was even more effective than $\alpha$-GalCer at preventing metastases of the B16 melanoma (92). These studies suggest that NKT cells do not need to directly kill the tumor cells, and they are not acting primarily as effectors, but instead they recruit and promote a response by downstream effectors in an IFN- $\gamma$-dependent manner. $\alpha$-GalCer-stimulated NKT cells may enhance 
both NK and CTL activity, and both effector cell types have been implicated in the $\alpha$-GalCer-induced antitumor response (87). NKT cell stimulation may also enhance tumor immunity by promoting APC activation and IL-12 production via CD40 ligand (CD40L) upregulation $(93,94)$. Thus, in many tumor models, NKT cells act to upregulate, rather than downregulate, the immune response, a type of regulation not observed with $\mathrm{CD} 25^{+} \mathrm{CD} 4^{+}$Tregs, which are uniformly suppressive.

It is important to emphasize that these studies support the concept that NKT cells can be induced to promote tumor rejection when using immunotherapeutic factors such as IL-12 or $\alpha$-GalCer. Whether this reflects the physiological activity of these cells is an important question, particularly in light of the fact that NKT cells are often associated with immunosuppression rather than immune aggression. Another model where NKT cells are clearly required for tumor rejection, this time in the absence of exogenous stimulation, is the rejection of methylcholanthreneinduced sarcomas in mice. In this model, sarcomas formed with far greater incidence in NKT cell-deficient $J \alpha 18^{-/-}$mice (95). Sarcoma cell lines also grew preferentially in NKT cell-deficient mice, and rejection could be mediated by adoptive transfer of purified NKT cells from WT donors (96). Similar findings were more recently reported for an experimental model of pulmonary sarcoma metastasis (38). As in $\alpha$-GalCer-induced NKT cell-mediated antitumor immunity, NKT cell-derived IFN- $\gamma$ was critical for sarcoma rejection, and both NK cells and CD8 T cells played an essential role as downstream effector cells (96).

Compounding the paradox of the differential immune activity mediated by NKT cells, in other mouse tumor models, NKT cells appear to play a suppressive role. Experimental tumors 15-12RM (97) and 4T1 (98) were rejected in CD1d $d^{-/-}$BALB/c mice but grew in WT BALB/c mice. The suppressive effect in the 15-12RM model was IL-13 dependent/IL-4 independent (97) and appeared to involve TGF- $\beta$ production by a Gr- $1^{+}$myeloid cell that led to impaired CTL responsiveness (99). In contrast, suppression of 4T1 tumor rejection in $C D 1 d^{-/-}$mice was not IL-13 dependent, which suggests the existence of multiple mechanisms by which CD1d-dependent cells can inhibit tumor rejection. It is noteworthy that depletion of $\mathrm{CD} 25^{+} \mathrm{CD} 4^{+}$Tregs in the 4T1 model also failed to restore tumor rejection. CD1ddependent $T$ cells have also been implicated in the UV-induced suppression of tumor rejection (100). It must be emphasized that while results from these models using $C D 1 d^{-/-}$mice suggest the involvement of type I "classical" V $\alpha 14-\mathrm{J} \alpha 18 \mathrm{NKT}$ cells, this should be confirmed using $J \alpha 18^{-/-}$mice, and it remains possible that nonclassical or V $\alpha 14-J \alpha 18$-negative but CD1d-dependent T cells, also known as type II NKT cells (1), are mediating tumor suppression in these models.

Numerical and/or functional deficiencies in NKT cells have been reported in association with some, but not all, types of human cancer (101-103), although it is not clear whether this is a cause or effect of the cancer. Furthermore, $\alpha$-GalCer-stimulated human NKT cells can kill or inhibit the growth of tumor cells in vitro $(101,104,105)$ and they can activate human NK cells through an IL-2-dependent mechanism (106), which highlights the potential of NKT cell stimulation for immunotherapy. $\alpha$-GalCer has been tested in phase I clinical trials, either by direct administration of the compound (107) or by administration of $\alpha$-GalCer-pulsed autologous DCs (108). The results of these studies showed that $\alpha$-GalCer therapy appears to be rela- tively safe at the doses used, with minimal side effects (107, 108). In both studies, biological responses were observed, including transiently reduced NKT cell and NK cell levels and transient increases in serum cytokines. The type of cytokine response appeared to vary between the 2 studies, with the most consistent results arising after therapy with $\alpha$-GalCer-pulsed DCs (108), where transient increases in serum IFN- $\gamma$ and IL-12, as well as signs of increased T and NK cell activation and enhanced NK cell cytotoxicity, were observed. Perhaps most encouragingly, the majority of patients in this study experienced tumor-associated inflammatory symptoms concurrent with $\alpha$-GalCer treatments, suggestive of increased immune activity (108). Similarly, in mouse studies, $\alpha$-GalCer-pulsed DCs were more effective at preventing melanoma metastases than the free compound (109).

\section{NKT cells and host defense}

NKT cells have been shown to participate in protection of mice from a variety of bacterial, viral, and protozoan parasites, although some of these results are controversial (reviewed in refs. 2 and 3). These controversies could have several sources, but an important one is genetic background. For example, in a cerebral malaria model system, NKT cells promoted pathogenesis in C57BL/ 6 mice, but they inhibited it in BALB/c mice (23). Similarly, the effect of NKT cells on the immune response to respiratory syncytial virus also differs between C57BL/6 and BALB/c mice (110).

Much less is known about the role of human NKT cells in infections, but there is a report of an 11-year-old girl with disseminated varicella infection after vaccination who was found to have a deficiency of NKT cells and no other immune defect (111).

In several instances where the mechanism of NKT cell action in host defense has been partially defined, IFN- $\gamma$ secretion is important (reviewed in ref. 3). This does not imply that NKT cells act purely as effectors in these cases, as the majority of this secretion might be due to NK cells that have been stimulated as a result of NKT cell activation, as has been seen in several studies $(71,72,91)$. As in other experimental systems, however, NKT cells can operate in either a stimulating or suppressive fashion. For example, analysis of $C D 1 d^{-/-}$and $J \alpha 18^{-/-}$mice indicates that clearance of herpes simplex virus type 1 is reduced in mice deficient for NKT cells (112). By contrast, NKT cells negatively regulate the response to lymphocytic choriomeningitis virus (113).

The extent to which NKT cells respond directly to microbial glycolipids is unclear, and in some instances, the recognition of microbes by NKT cells may be indirect. One study suggested that salmonella infection may lead to an altered environment, where NKT cells are stimulated by inflammatory cytokines in combination with CD1d-mediated presentation of autologous ligands, induced in response to infection (114). This is consistent with the role played by NKT cells in viral infections, because viruses do not contain antigenic glycolipids, although they may contain glycolipids produced by host cells. In another study, NKT cells were required for the formation of granulomatous lesions following injection of glycolipids derived from mycobacteria (115). The recruitment of NKT cells to granulomas does not require recognition of cognate antigen by the NKT cell TCR, however, as it can occur even when these $T$ cells are adoptively transferred to $C D 1 d^{-/-}$mice (116). This recruitment to inflammatory sites is likely to be driven by cytokines, such as TNF (116), independently of TCR stimulation. Direct evidence for NKT cell recognition 
A

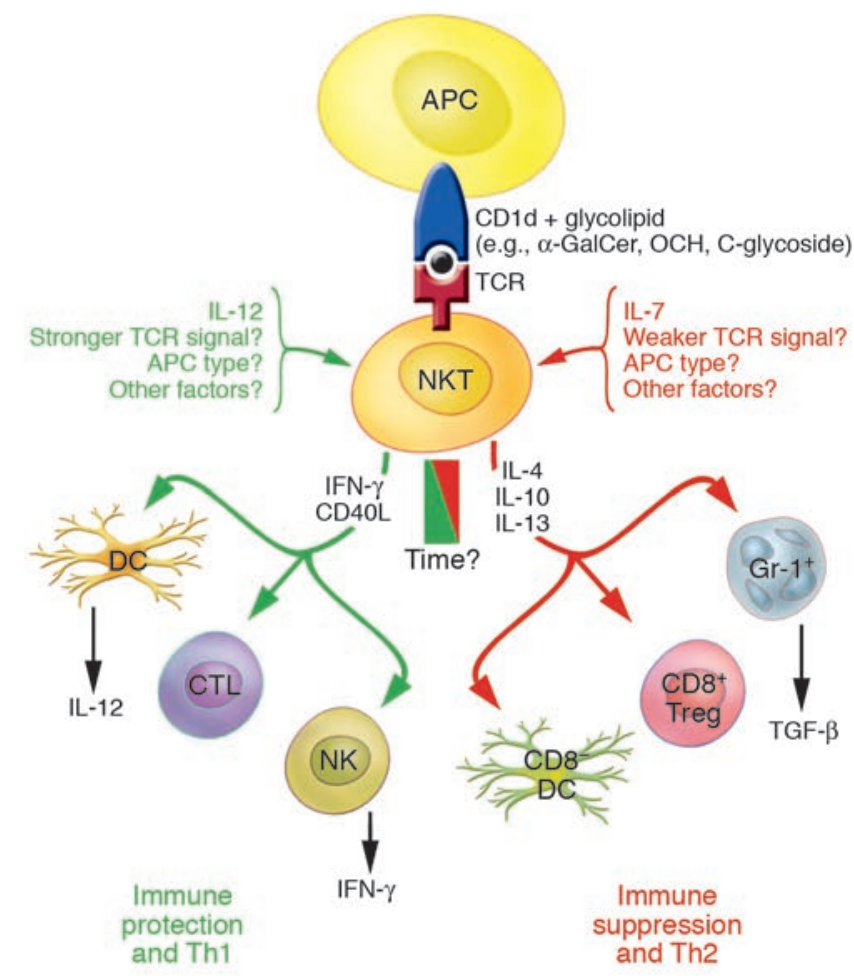

B

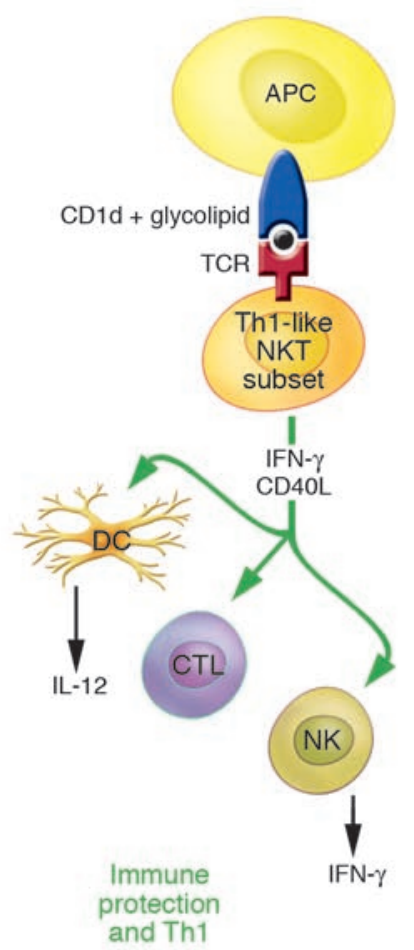

Figure 1

Schematic outline as to how NKT cells influence the immune response in either a Th1 or Th2 direction. (A) Environmental model. The green side shows factors supporting the Th1 responsiveness by NKT cells, while the red side shows factors supporting Th2 responsiveness by these cells. The environment in which NKT cells are found, including the type of cytokines and APCs and the strength of antigen-mediated TCR stimulation they receive, determines the cytokine output. Upregulation of cell-surface CD40L by NKT cells stimulates IL-12 release by DCs, which work together with NKT cell-derived IFN- $\gamma$ to stimulate Th1 responses. IL-7 has been reported to enhance Th2 cytokine production by NKT cells, while cell-surface molecules that mediate immune suppression or Th2 deviation by these cells have not been identified. Time after stimulation may also be an important factor, as NKT cells also tend to produce Th1- and Th2-like cytokines in the short term (1-3 hours) following stimulation but may then switch to a Th1 output, which can last for several days. (B) Subset model. Preexisting subsets of NKT cells are programmed to produce different ratios of Th1/Th2 cytokines, as reported for CD4- and CD4+ human NKT cells. In this case, the influence of NKT cells on the immune response would depend on the prevalence and/or relative involvement of these 2 subsets. The 2 models are not necessarily mutually exclusive.

of microbial ligands was presented in a very recent report in which they were shown to recognize and respond to mycobacterial phosphatidylinositol tetramannoside in conjunction with CD1d (117), although this involved only a minority of the NKT cells. The role of NKT cells in infection is clearly an exciting area of investigation with obvious clinical potential. The possibility that these cells can enhance immunity to microbial organisms, via either direct recognition or indirect mechanisms, makes them important targets for future vaccine design.

\section{NKT cells in allergy and inflammation}

Several recent studies have implicated mouse NKT cells in allergic and inflammatory responses. In each case, the degree to which NKT cells serve as regulators, as opposed to effectors of immune-mediated damage, remains to be determined. The very small numbers of NKT cells that can be isolated from target organs, such as the lungs, suggest these cells are acting primarily in a regulatory capacity. The best-studied system is airway hypersensitivity, which can be induced in mice by sen- sitization with antigen followed by intranasal antigen challenge. The first study of the role of NKT cells in this process, using OVA as a model sensitizing antigen, compared NKT cell-deficient $J \alpha 18^{-/-}$mice with WT mice and failed to reveal a role for NKT cells in OVA-induced airway hypersensitivity (118). However, 2 more recent studies indicated that NKT cells are required for the induction of airway sensitivity $(119,120)$. Transfer of NKT cells to J $\alpha 18^{-/-}$mice restored susceptibility and enabled the demonstration that either $I L-4^{-/-}$or $I L-13^{-/-}$NKT cells were competent to do this, while NKT cells deficient for both cytokine genes were not (120). These findings are not confined to OVA immunization, as $C D 1 d^{-/-}$mice also showed a decreased response to the ragweed allergen when airway eosinophilia, mucus production, and anti-ragweed $\operatorname{IgE}$ were measured (121). Despite this, in humans, the frequency of $V \alpha 24-$ expressing $\mathrm{CD} 161^{+}$peripheral blood lymphocytes was equivalent in atopic and non-atopic individuals (122), which indicates that caution is required when extrapolating from animal models to humans. While at face value, this might suggest that NKT 
cells are not involved in human atopy, this may again reflect the problem that NKT cell levels in peripheral blood do not necessarily mirror those in other tissues. A more meaningful assessment of NKT cells in human airway hyperresponsiveness might come from studying these cells in bronchoalveolar lavage samples. Considering the Th2 cytokine production by NKT cells in humans, and abundant data in support of their role in allergy in murine models, we strongly feel that further investigation into NKT cells in human allergy is warranted.

Mouse NKT cells have been implicated in several other contexts where stimulation of the immune system leads to inflammation or pathology. Contact hypersensitivity in mice has been shown to require IL-4 production by NKT cells (123). This IL-4 acts rapidly in a cascade that involves activation of B-1 B cells and mast cells, which leads to vascular permeability and the recruitment of effector $\mathrm{T}$ cells to the site where antigen is located in the skin (123). In this case, therefore, it has been established that NKT cell IL-4 is playing a regulatory role in terms of modulating the activity of subsets of B lymphocytes as well as mast cells. Immunity and inflammation mediated by the innate and adaptive immune systems have been implicated in the pathogenesis of atherosclerosis. Interestingly, in atherosclerosis-prone apoE $E^{-/-}$mice crossed with $C D 1 d^{-/-}$mice, lesion sizes in the arteries were decreased compared with control apoE $E^{-/-}$mice (124). Furthermore, administration of $\alpha$-GalCer increased lesion size in $a p o E^{-/-}$mice that were CD1d positive. These results suggest that NKT cells exacerbate atherosclerosis, although no mechanism was defined.

\section{Which way will they go?}

Although NKT cells potentially can act as effectors, as exemplified by their cytotoxic activity, it is likely that regulatory function reflects their true physiologic role. This is consistent with their small numbers, the known regulatory influences of the cytokines they secrete, and their documented close interactions with DCs and other cell types that in turn influence adaptive immunity. In thinking of NKT cells as regulators, however, it is necessary to abandon the classic concept of regulation as equivalent to suppression, because clearly the effects of NKT cells are not mostly suppressive. A question of central importance is how NKT cells "decide" which way to go - that is, whether to promote or suppress immune responses. Several possibilities are summarized in Figure 1.

(a) NKT cell subsets may be functionally distinct, such that some phenotypic subsets (e.g., $\mathrm{CD}^{+}{ }^{+}$or $\mathrm{CD}^{-}$) or NKT cells in different sites (e.g., thymus or liver) might be better at suppressing immune responses, while others are better at promoting immune responses. There is some evidence for the existence of functionally distinct NKT cell subsets, defined as $\mathrm{CD}^{+}$or $\mathrm{CD}^{-}$, in humans $(12,32)$. Human $\mathrm{CD}^{+}$NKT cells produce a higher Th2/Th 1 cytokine ratio and exhibit a distinct pattern of chemokine receptors and cytotoxic molecules, compared with CD4- NKT cells. However, there is little evidence for such a distinction in mice.

(b) NKT cells might preferentially produce pro- or anti-inflammatory cytokines, depending on the type of signals these cells receive. For example, IL-12 or anti-NK1.1 crosslinking has been shown to preferentially induce IFN- $\gamma$ production by NKT cells, while IL-7 promotes IL-4 production (2). These cytokine signals might be integrated with TCR signals. Consistent with this, puls- ing DCs with $\alpha$-GalCer has been reported to prolong and enhance IFN- $\gamma$ production (109), perhaps by providing the appropriate cytokines or costimulatory signals to augment Th1 responses.

(c) Different types of TCR stimulation might alter the cytokine profile. In support of this, the OCH analog of $\alpha$-GalCer has been found to preferentially induce Th2 responses $(68,70)$, while the C-glycoside preferentially induces Th1 cytokines (92). These responses may be a direct reflection of cytokine production by the NKT cells themselves or differences in the impact of NKT cells on cytokine production by downstream cells. In one sense, this may not be the most important consideration, as the net effect remains a differentially modulated immune response. As new NKT cell antigens are starting to emerge, it will be very important to assess the impact that these have on NKT cell function.

(d) NKT cells might typically produce the same array of factors when stimulated, but the impact of these factors is interpreted differently in different models, thus taking the "decision" away from the NKT cells themselves. This is supported by studies in mice showing that nearly all NKT cells produce both IFN- $\gamma$ and IL- 4 immediately after $\alpha$-GalCer injection $(9,29,30)$, and indeed, they contain pre-formed mRNA for these cytokines (29), which would explain their rapid production of such proteins upon stimulation. The types of cytokines produced by these cells over the longer term is less clear, although one study suggested that IL-4 production by NKT cells ceases within hours of stimulation, while IFN- $\gamma$ production is sustained for $2-3$ days (31). This raises the possibility that the impact of NKT cells depends on the temporal nature of their encounter with other immune cells.

While these 4 possibilities seem quite distinct, there is some evidence to support each one, and it must be emphasized that they are not necessarily mutually exclusive. Further investigation with the possibilities in mind is required to determine which factors predominate in the various diseases that are known to be influenced by NKT cells.

\section{Future clinical potential for NKT cell-based immunotherapy}

The fact that NKT cells represent highly potent immunoregulatory cells with a conserved specificity makes them very attractive targets for immunotherapy. However, there is much to be learned before these cells can be safely and effectively manipulated in the clinic, due to their poorly understood and dichotomous behavior. There are 2 classes of NKT cell-mediated immune regulation: those that require exogenous stimulatory factors, such as $\alpha$-GalCer or IL-12, and those where NKT cells respond without intentional stimulation - that is, they simply need to be present. In both classes, the NKT cell response can either promote or suppress the associated immune response, and this is not always to the benefit of the host.

The identification and characterization of the various molecules/signals that lead to NKT cell activation is obviously an important goal that should allow more precise manipulation of NKT cell activity. Furthermore, techniques that allow expansion of NKT cells or subsets thereof, either in vivo or in vitro followed by reinjection, may be sufficient to manipulate immune responses in a clinical setting. This could be an important stratagem if NKT cell regulatory activity does find a clinical use, as the frequency of these cells in humans is both highly variable and generally lower than that in mice. Ultimately, a greater understanding of the 
nature of distinct NKT cell subsets and/or the influence of different CD1d-binding NKT cell ligands, microenvironmental factors, and APCs should enable us to selectively manipulate NKT cells to go one way or the other.

\section{Acknowledgments}

The authors wish to thank the many colleagues in the NKT cell and related fields for stimulating discussions that were helpful in writing this review and Stuart Berzins for critically reading this manuscript. D.I. Godfrey was supported by a National Health and Medical Research Council Fellowship and Program Grant,
ADCORP/Diabetes Australia, and Rothschild Australia. M. Kronenberg is supported by an NIH RO1 grant (CA52511).

Address correspondence to: Dale Godfrey, Department of Microbiology and Immunology, University of Melbourne, Parkville, Victoria 3010, Australia. Phone: 61-3-83448631; Fax: 61-3-93471540; E-mail: godfrey@unimelb.edu.au. Or to: Mitchell Kronenberg, Division of Developmental Immunology, La Jolla Institute for Allergy and Immunology, 10355 Science Center Drive, San Diego, California 92121, USA. Phone: (858) 678-4540; Fax: (858) 678-4595; E-mail:mitch@liai.org.
1. Godfrey, D.I., MacDonald, H.R., Kronenberg, M., Smyth, M.J., and Van Kaer, L. 2004. NKT cells: what's in a name? Nat. Rev. Immunol. 4:231-237.

2. Godfrey, D.I., Hammond, K.J., Poulton, L.D., Smyth, M.J., and Baxter, A.G. 2000. NKT cells: facts, functions and fallacies. Immunol. Today. 21:573-583.

3. Kronenberg, M., and Gapin, L. 2002. The unconventional lifestyle of NKT cells. Nat. Rev. Immunol. 2:557-568

4. Dellabona, P., Padovan, E., Casorati, M., Brockhaus, M., and Lanzavecchia, A. 1994. An invariant $\mathrm{V} \alpha 24-\mathrm{J} \alpha \mathrm{Q} / \mathrm{V} \beta 11 \mathrm{~T}$ cell receptor is expressed in all individuals by clonally expanded CD4-8- T cells J. Exp. Med. 180:1171-1176.

5. Porcelli, S., Yockey, C.E., Brenner, M.B., and Balk, S.P. 1993. Analysis of T cell antigen receptor (TCR) expression by human peripheral blood CD4-8- alpha/beta $T$ cells demonstrates preferential use of several Vbeta genes and an invariant TCR alpha chain. J. Exp. Med. 178:1-16.

6. Brossay, L., et al. 1998. Cd1d-mediated recognition of an alpha-galactosylceramide by natural killer $t$ cells is highly conserved through mammalian evolution. J. Exp. Med. 188:1521-1528.

7. MacDonald, H.R. 2002. Development and selection of NKT cells. Curr. Opin. Immunol. 14:250-254.

8. Hammond, K.J.L., et al. 1999. NKT cells are phenotypically and functionally diverse. Eur. $J$. Immunol. 29:3768-3781.

9. Matsuda, J.L., et al. 2000. Tracking the response of natural killer T cells to a glycolipid antigen using CD1d tetramers. J. Exp. Med. 192:741-754.

10. Eberl, G., et al. 1999. Tissue-specific segregation of CD1d-dependent and CD1d-independent NK T cells. J. Immunol. 162:6410-6419.

11. Ishihara, S., et al. 1999. CD8 ${ }^{+}$NKR-P1A ${ }^{+}$T cells preferentially accumulate in human liver. Eur. $J$. Immunol. 29:2406-2413.

12. Gumperz, J.E., Miyake, S., Yamamura, T., and Brenner, M.B. 2002. Functionally distinct subsets of CD1d-restricted natural killer T cells revealed by CD1d tetramer staining. J. Exp. Med. 195:625-636.

13. Prussin, C., and Foster, B. 1997. TCR V-alpha-24 and V-beta-11 coexpression defines a human NK1 T cell analog containing a unique Th0 subpopulation. J. Immunol. 159:5862-5870.

14. Exley, M.A., et al. 2002. Cutting edge: compartmentalization of Th1-like noninvariant CD1d reactive $T$ cells in hepatitis $C$ virus-infected liver. J. Immunol. 168:1519-1523.

15. Kenna, T., et al. 2003. NKT cells from normal and tumor-bearing human livers are phenotypically and functionally distinct from murine NKT cells. J. Immunol. 171:1775-1779.

16. Gumperz, J.E., and Brenner, M.B. 2001. CD1-specific T cells in microbial immunity [review]. Curr Opin. Immunol. 13:471-478.

17. Hayakawa, Y., Godfrey, D.I., and Smyth, M.J. 2004. Alpha-galactosylceramide: potential immunomodulatory activity and future application. Curr. Med. Chem. 11:241-252.

18. Hong, S.M., et al. 1999. Lipid antigen presenta- tion in the immune system: lessons learned from CD1d knockout mice. Immunol. Rev. 169:31-44.

19. Sidobre, S., et al. 2002. The V alpha 14 NKT cell TCR exhibits high-affinity binding to a glycolipid/ CD1d complex. J. Immunol. 169:1340-1348.

20. Hammond, K.J., et al. 2001. CD1d-restricted NKT cells: an interstrain comparison. J. Immunol. 167:1164-1173.

21. Benlagha, K., Weiss, A., Beavis, A., Teyton, L., and Bendelac, A. 2000. In vivo identification of glycolipid antigen-specific T cells using fluorescent CD1d tetramers. J. Exp. Med. 191:1895-1903.

22. Wu, D.Y., Segal, N.H., Sidobre, S., Kronenberg, M., and Chapman, P.B. 2003. Cross-presentation of Disialoganglioside GD3 to Natural Killer T Cells. J. Exp. Med. 198:173-181.

23. Hansen, D.S., Siomos, M.A., Buckingham, L. Scalzo, A.A., and Schofield, L. 2003. Regulation of murine cerebral malaria pathogenesis by CD1d-restricted NKT cells and the natural killer complex. Immunity. 18:391-402.

24. Schofield, L., et al. 1999. CD1d-restricted immunoglobulin $\mathrm{G}$ formation to GPI-anchored antigens mediated by NKT cells. Science. 283:225-229.

25. Rauch, J., et al. 2003. Structural features of the acyl chain determine self-phospholipid antigen recognition by a CD1d-restricted invariant NKT (iNKT) cell. J. Biol. Chem. 278:47508-47515.

26. Ortaldo, J.R., et al. 2004. Dissociation of NKT stimulation, cytokine induction, and NK activation in vivo by the use of distinct TCR-binding ceramides. J. Immunol. 172:943-953.

27. Smyth, M.J., and Godfrey, D.I. 2000. NKT cells and tumor immunity--a double-edged sword. Nat. Immunol. 1:459-460.

28. Wilson, S.B., and Delovitch, T.L. 2003. Janus-like role of regulatory iNKT cells in autoimmune disease and tumour immunity. Nat. Rev. Immunol. 3:211-222.

29. Matsuda, J.L., et al. 2003. Mouse V alpha $14 \mathrm{i}$ natural killer $\mathrm{T}$ cells are resistant to cytokine polarization in vivo. Proc. Natl. Acad. Sci. U. S. A. 100:8395-8400.

30. Stetson, D.B., et al. 2003. Constitutive cytokine mRNAs mark natural killer (NK) and NK T cells poised for rapid effector function. J. Exp. Med. 198:1069-1076

31. Crowe, N.Y., et al. 2003. Glycolipid antigen drives rapid expansion and sustained cytokine production by NKT cells. J. Immunol. 171:4020-4027.

32. Lee, P.T., Benlagha, K., Teyton, L., and Bendelac, A. 2002. Distinct functional lineages of human $\mathrm{V}$ alpha-24 natural killer T cells. J. Exp. Med. 195:637-641.

33. Wang, Y., Goldschneider, I., O'Rourke, J., and Cone, R.E. 2001. Blood mononuclear cells induce regulatory NK T thymocytes in anterior chamber-associated immune deviation. J. Leukoc. Biol. 69:741-746.

34. Wang, Y.F., et al. 1997. Direct thymic involvement in anterior chamber-associated immune deviation - evidence for a nondeletional mechanism of centrally induced tolerance to extrathymic anti- gens in adult mice. J. Immunol. 158:2150-2155.

35. Sonoda, K.H., Exley, M., Snapper, S., Balk, S.P., and Stein-Streilein, J. 1999. CD1-reactive natural killer $\mathrm{T}$ cells are required for development of systemic tolerance through an immune-privileged site. J. Exp. Med. 190:1215-1225.

36. Sonoda, K.H., et al. 2001. NK T cell-derived IL-10 is essential for the differentiation of antigenspecific $\mathrm{T}$ regulatory cells in systemic tolerance. J. Immunol. 166:42-50.

37. Nakamura, T., et al. 2003. CD4(+) NKT cells, but not conventional CD4(+) $\mathrm{T}$ cells, are required to generate efferent CD8(+) T regulatory cells following antigen inoculation in an immune-privileged site. J. Immunol. 171:1266-1271.

38. Nishikawa, H., et al. 2003. CD4+ CD25+ T cells responding to serologically defined autoantigens suppress antitumor immune responses. Proc. Natl. Acad. Sci. U. S. A. 100:10902-10906.

39. Azuma, T., Takahashi, T., Kunisato, A., Kitamura, T., and Hirai, H. 2003. Human CD4+ CD25+ regulatory $\mathrm{T}$ cells suppress NKT cell functions. Cancer Res. 63:4516-4520.

40. Sonoda, K.H., Taniguchi, M., and Stein-Streilein, J. 2002. Long-term survival of corneal allografts is dependent on intact CD1d-reactive NKT cells. J. Immunol. 168:2028-2034.

41. Seino, K.-I., et al. 2001. Requirement for natural killer T (NKT) cells in the induction of allograft tolerance. Proc. Natl. Acad. Sci. U. S. A. 98:2577-2581.

42. Higuchi, M., et al. 2002. Immune tolerance to combined organ and bone marrow transplants after fractionated lymphoid irradiation involves regulatory $\mathrm{NK} \mathrm{T}$ cells and clonal deletion. J. Immunol. 169:5564-5570.

43. Ikehara, Y., et al. 2000. CD4 ${ }^{+} \mathrm{V} \alpha 14$ natural killer $\mathrm{T}$ cells are essential for acceptance of rat islet xenografts in mice. J. Clin. Invest. 105:1761-1767.

44. Zeng, D.F., et al. 1999. Bone marrow NK1.1' and $\mathrm{NK} 1.1^{+} \mathrm{T}$ cells reciprocally regulate acute graft versus host disease. J. Exp. Med. 189:1073-1081.

45. Lan, F., et al. 2001. Predominance of NK1.1+TCR alpha beta+ or DX5+TCR alpha beta+ T cells in mice conditioned with fractionated lymphoid irradiation protects against graft-versus-host disease: "natural suppressor" cells. J. Immunol. 167:2087-2096.

46. Ito, K., et al. 2000. Involvement of decidual V alpha 14 NKT cells in abortion. Proc. Natl. Acad. Sci. U. S. A. 97:740-744

47. Hammond, K.J., and Godfrey, D.I. 2002. NKT cells: potential targets for autoimmune disease therapy? Tissue Antigens. 59:353-363.

48. Hammond, K.J., and Kronenberg, M. 2003. Natural killer T cells: natural or unnatural regulators of autoimmunity? Curr. Opin. Immunol. 15:683-689.

49. Baxter, A.G., Kinder, S.J., Hammond, H.J.L., Scollay, R., and Godfrey, D.I. 1997. Association between $\alpha \beta \mathrm{TCR}^{+} \mathrm{CD} 4-\mathrm{CD} 8-\mathrm{T}$ cell deficiency and IDDM in NOD/Lt mice. Diabetes. 46:572-582.

50. Hammond, K.J.L., et al. 1998. Alpha/beta-T cell receptor $(\mathrm{TCR})^{+} \mathrm{CD} 4-\mathrm{CD} 8-(\mathrm{NKT})$ thymocytes prevent insulin-dependent diabetes mellitus in 
nonobese diabetic (NOD/Lt) mice by the influence of interleukin (IL)-4 and/or IL-10. J. Exp. Med. 187:1047-1056.

51. Lehuen, A., et al. 1998. Overexpression of natural killer T cells protects V $\alpha 14-J \alpha 281$ transgenic nonobese diabetic mice against diabetes. J. Exp. Med. 188:1831-1839.

52. Poulton, L.D., et al. 2001. Cytometric and functional analyses of NK and NKT cell deficiencies in NOD mice. Int. Immunol. 13:887-896.

53. Hong, S., et al. 2001. The natural killer T-cell ligand alpha-galactosylceramide prevents autoimmune diabetes in non-obese diabetic mice. Nat. Med. 7:1052-1056.

54. Gombert, J.M., et al. 1996. Early quantitative and functional deficiency of $\mathrm{NK} 1^{+}$-like thymocytes in the NOD mouse. Eur. J. Immunol. 26:2989-2998.

55. Wang, B., Geng, Y.B., and Wang, C.R. 2001. CD1-restricted NKT cells protect nonobese diabetic mice from developing diabetes. J. Exp. Med. 194:313-320.

56. Sharif, S., et al. 2001. Activation of natural killer T cells by alpha-galactosylceramide treatment prevents the onset and recurrence of autoimmune Type 1 diabetes. Nat. Med. 7:1057-1062.

57. Naumov, Y.N., et al. 2001. Activation of CD1drestricted $\mathrm{T}$ cells protects NOD mice from developing diabetes by regulating dendritic cell subsets. Proc. Natl. Acad. Sci. U. S. A. 98:13838-13843.

58. Beaudoin, L., Laloux, V., Novak, J., Lucas, B., and Lehuen, A. 2002. NKT cells inhibit the onset of diabetes by impairing the development of pathogenic $\mathrm{T}$ cells specific for pancreatic beta cells. Immunity. 17:725-736.

59. Wilson, S.B., et al. 1998. Extreme TH1 bias of invariant V-alpha-24J-alpha-Q T cells in type 1 diabetes. Nature. 391:177-181.

60. Kukreja, A., et al. 2002. Multiple immuno-regulatory defects in type-1 diabetes. J. Clin. Invest. 109:131-140. doi:10.1172/JCI200213605.

61. Oikawa, Y., et al. 2002. High frequency of valpha24(+) vbeta11(+) T-cells observed in type 1 diabetes. Diabetes Care. 25:1818-1823.

62. Lee, P.T., et al. 2002. Testing the NKT cell hypothesis of human IDDM pathogenesis. J. Clin. Invest. 110:793-800. doi:10.1172/JCI200215832.

63. Berzins, S.P., et al. 2004. Systemic NKT cell deficiency in NOD mice is not detected in peripheral blood: implications for human studies. Immunol. Cell Biol. 82:247-252.

64. Furlan, R., et al. 2003. Activation of invariant NKT cells by alphaGalCer administration protects mice from MOG35-55-induced EAE: critical roles for administration route and IFN-gamma. Eur. J. Immunol. 33:1830-1838.

65. Jahng, A.W., et al. 2001. Activation of natural killer T cells potentiates or prevents experimental autoimmune encephalomyelitis. J. Exp. Med. 194:1789-1799.

66. Singh, A.K., et al. 2001. Natural killer T cell activation protects mice against experimental autoimmune encephalomyelitis. J. Exp. Med. 194:1801-1811.

67. Pal, E., et al. 2001. Costimulation-dependent modulation of experimental autoimmune encephalomyelitis by ligand stimulation of V alpha $14 \mathrm{NK}$ T cells. J. Immunol. 166:662-668.

68. Miyamoto, K., Miyake, S., and Yamamura, T. 2001. A synthetic glycolipid prevents autoimmune encephalomyelitis by inducing TH2 bias of natural killer T cells. Nature. 413:531-534.

69. Chiba, A., et al. 2004. Suppression of collageninduced arthritis by natural killer $\mathrm{T}$ cell activation with $\mathrm{OCH}$, a sphingosine-truncated analog of alpha-galactosylceramide. Arthritis Rheum. 50:305-313.

70. Stanic, A.K., et al. 2003. Another view of T cell antigen recognition: cooperative engagement of glycolipid antigens by Va14Ja18 natural TCR.
J. Immunol. 171:4539-4551.

71. Hayakawa, Y., et al. 2001. Critical contribution of IFN-gamma and NK cells, but not perforinmediated cytotoxicity, to anti-metastatic effect of alpha-galactosylceramide. Eur. J. Immunol. 31:1720-1727.

72. Carnaud, C., et al. 1999. Cutting edge: crosstalk between cells of the innate immune system: NKT cells rapidly activate NK cells. J. Immunol. 163:4647-4650.

73. Mars, L.T., et al. 2002. Cutting edge: V alpha 14-J alpha 281 NKT cells naturally regulate experimental autoimmune encephalomyelitis in nonobese diabetic mice. J. Immunol. 168:6007-6011.

74. Araki, M., et al. 2003. Th2 bias of CD4+ NKT cells derived from multiple sclerosis in remission. Int. Immunol. 15:279-288.

75. Takeda, K., and Dennert, G. 1993. The development of autoimmunity in C57BL/6 lpr mice correlates with the disappearance of natural killer type-1 positive cells: evidence for their suppressive action on bone marrow stem cell proliferation, B cell immunoglobulin secretion, and autoimmune symptoms. J. Exp. Med. 177:155-164.

76. Mieza, M.A., et al. 1996. Selective reduction of V-alpha-14+ NKT cells associated with disease development in autoimmune-prone mice. J. Immunol. 156:4035-4040.

77. Yang, J.Q., et al. 2003. Immunoregulatory role of CD1d in the hydrocarbon oil-induced model of lupus nephritis. J. Immunol. 171:2142-2153.

78. Yang, J.Q., et al. 2004. CD1d deficiency exacerbates inflammatory dermatitis in MRL-lpr/lpr mice. Eur. J. Immunol. 34:1723-1732.

79. Chan, O.T., et al. 2001. Deficiency in beta(2)microglobulin, but not CD1, accelerates spontaneous lupus skin disease while inhibiting nephritis in MRL-Fas(lpr) nice: an example of disease regulation at the organ level. J. Immunol. 167:2985-2990.

80. Yang, J.Q., et al. 2003. Repeated alpha-galactosylceramide administration results in expansion of NK T cells and alleviates inflammatory dermatitis in MRL-lpr/lpr mice. J. Immunol. 171:4439-4446.

81. Zeng, D., Liu, Y., Sidobre, S., Kronenberg, M., and Strober, S. 2003. Activation of natural killer T cells in NZB/W mice induces Th1-type immune responses exacerbating lupus. J. Clin. Invest. 112:1211-1222. doi:10.1172/JCI200317165.

82. Zeng, D., Lee, M.K., Tung, J., Brendolan, A., and Strober, S. 2000. Cutting edge: a role for CD1 in the pathogenesis of lupus in NZB/NZW mice. J. Immunol. 164:5000-5004.

83. Saubermann, L.J., et al. 2000. Activation of natural killer $\mathrm{T}$ cells by alpha-galactosylceramide in the presence of CD1d provides protection against colitis in mice. Gastroenterology. 119:119-128.

84. Heller, F., Fuss, I.J., Nieuwenhuis, E.E., Blumberg, R.S., and Strober, W. 2002. Oxazolone colitis, a Th2 colitis model resembling ulcerative colitis, is mediated by IL-13-producing NK-T cells. Immunity. 17:629-638.

85. van der Vliet, H.J., et al. 2001. Circulating $\mathrm{V}($ alpha24+) Vbeta11+ NKT cell numbers are decreased in a wide variety of diseases that are characterized by autoreactive tissue damage. Clin. Immunol. 100:144-148.

86. Kita, H., et al. 2002. Quantitation and phenotypic analysis of natural killer $T$ cells in primary biliary cirrhosis using a human CD1d tetramer. Gastroenterology. 123:1031-1043.

87. Smyth, M.J., Crowe, N.Y., Takeda, K., Yagita, H., and Godfrey, D.I. 2002. NKT cells - conductors of tumor immunity? Curr. Opin. Immunol. 14:165-171.

88. Cui, J., et al. 1997. Requirement for Valpha14 NKT cells in IL-12-mediated rejection of tumors. Science. 278:1623-1626.

89. Kawano, T., et al. 1997. CD1d-restricted and TCR- mediated activation of V $\alpha 14$ NKT cells by glycosylceramides. Science. 278:1626-1629.

90. Hayakawa, Y., Rovero, S., Forni, G., and Smyth, M.J. 2003. Alpha-galactosylceramide (KRN7000) suppression of chemical- and oncogene-dependent carcinogenesis. Proc. Natl. Acad. Sci. U. S. A. 100:9464-9469.

91. Smyth, M.J., et al. 2002. Sequential production of interferon-gamma by NK1.1(+) T cells and natural killer cells is essential for the antimetastatic effect of alpha-galactosylceramide. Blood. 99:1259-1266.

92. Schmieg, J., Yang, G., Franck, R.W., and Tsuji, M. 2003. Superior protection against malaria and melanoma metastases by a C-glycoside analogue of the natural killer T cell ligand alpha-Galactosylceramide. J. Exp. Med. 198:1631-1641.

93. Kitamura, H., et al. 1999. The natural killer T (NKT) cell ligand alpha-galactosylceramide demonstrates its immunopotentiating effect by inducing interleukin (IL)-12 production by dendritic cells and IL-12 receptor expression on NKT cells. J. Exp. Med. 189:1121-1127.

94. Fujii, S., Shimizu, K., Smith, C., Bonifaz, L., and Steinman, R.M. 2003. Activation of natural killer $T$ cells by alpha-galactosylceramide rapidly induces the full maturation of dendritic cells in vivo and thereby acts as an adjuvant for combined CD4 and CD8 T cell immunity to a coadministered protein. J. Exp. Med. 198:267-279.

95. Smyth, M.J., et al. 2000. Differential tumor surveillance by natural killer (NK) and NKT cells. J. Exp. Med. 191:661-668.

96. Crowe, N.Y., Smyth, M.J., and Godfrey, D.I. 2002. A critical role for natural killer $T$ cells in immunosurveillance of methylcholanthreneinduced sarcomas. J. Exp. Med. 196:119-127.

97. Terabe, M., et al. 2000. NKT cell-mediated repression of tumor immunosurveillance by IL-13 and the IL-4R-STAT6 pathway. Nat. Immunol. 1:515-520.

98. Ostrand-Rosenberg, S., et al. 2002. Resistance to metastatic disease in STAT6-deficient mice requires hemopoietic and nonhemopoietic cells and is IFN-gamma dependent. J. Immunol. 169:5796-5804.

99. Terabe, M., et al. 2003. Transforming growth factor-beta production and myeloid cells are an effector mechanism through which CD1d-restricted $\mathrm{T}$ cells block cytotoxic $\mathrm{T}$ lymphocyte-mediated tumor immunosurveillance: abrogation prevents tumor recurrence. J. Exp. Med. 198:1741-1752.

100. Moodycliffe, A.M., Nghiem, D., Clydesdale, G., and Ullrich, S.E. 2000. Immune suppression and skin cancer development: regulation by NKT cells. Nat. Immunol. 1:521-525.

101. Motohashi, S., et al. 2002. Preserved IFN-alpha production of circulating $\mathrm{V}$ alpha 24 NKT cells in primary lung cancer patients. Int. J. Cancer. 102:159-165.

102.Ali Tahir, S.M., et al. 2001. Loss of IFN-gamma production by invariant NK T cells in advanced cancer. J. Immunol. 167:4046-4050.

103. Dhodapkar, M.V., et al. 2003. A reversible defect in natural killer $\mathrm{T}$ cell function characterizes the progression of premalignant to malignant multiple myeloma. J. Exp. Med. 197:1667-1676.

104.Kawano, T., et al. 1999. Antitumor cytotoxicity mediated by ligand-activated human V alpha24 NKT cells. Cancer Res. 59:5102-5105.

105. Kikuchi, A., et al. 2001. In vitro anti-tumour activity of alpha-galactosylceramide-stimulated human invariant Valpha24+NKT cells against melanoma. Br. J. Cancer. 85:741-746.

106. Metelitsa, L.S., et al. 2001. Human NKT cells mediate antitumor cytotoxicity directly by recognizing target cell CD1d with bound ligand or indirectly by producing IL-2 to activate NK cells. J. Immunol. 167:3114-3122. 
107. Giaccone, G., et al. 2002. A phase I study of the natural killer T-cell ligand alpha-Galactosylceramide (KRN7000) in patients with solid tumors. Clin. Cancer Res. 8:3702-3709.

108.Nieda, M., et al. 2004. Therapeutic activation of V\{alpha\}24+V\{beta\}11+ NKT cells in human subjects results in highly coordinated secondary activation of acquired and innate immunity. Blood. 103:383-389.

109.Fujii, S., Shimizu, K., Kronenberg, M., and Steinman, R.M. 2002. Prolonged IFN-gamma-producing NKT response induced with alpha-galactosylceramide-loaded DCs. Nat. Immunol. 3:867-874.

110.Johnson, T.R., Hong, S.M., Van Kaer, L., Koezuka, Y., and Graham, B.S. 2002. NK T cells contribute to expansion of CD8 $(+) \mathrm{T}$ cells and amplification of antiviral immune responses to respiratory syncytial virus. J. Virol. 76:4294-4303.

111.Levy, O., et al. 2003. Disseminated varicella infection due to the vaccine strain of varicella-zoster virus, in a patient with a novel deficiency in natural killer T cells. J. Infect. Dis. 188:948-953.

112.Grubor-Bauk, B., Simmons, A., Mayrhofer, G. and Speck, P.G. 2003. Impaired clearance of herpes simplex virus type 1 from mice lacking CD $1 \mathrm{~d}$ or NKT cells expressing the semivariant $\mathrm{V}$ alpha
14-J alpha 281 TCR. J. Immunol. 170:1430-1434.

113.Roberts, T.J., Lin, Y., Spence, P.M., Van Kaer, L., and Brutkiewicz, R.R. 2004. CD1d1-dependent control of the magnitude of an acute antiviral immune response. J. Immunol. 172:3454-3461.

114.Brigl, M., Bry, L., Kent, S.C., Gumperz, J.E., and Brenner, M.B. 2003. Mechanism of CD1d-restricted natural killer $\mathrm{T}$ cell activation during microbial infection. Nat. Immunol. 4:1230-1237.

115.Apostolou, I., et al. 1999. Murine natural killer $\mathrm{T}$ (NKT) cells contribute to the granulomatous reaction caused by mycobacterial cell walls. Proc. Natl. Acad. Sci. U. S. A. 96:5141-5146.

116.Mempel, M., et al. 2002. Natural killer T cells restricted by the monomorphic MHC class $1 \mathrm{~b}$ CD1d1 molecules behave like inflammatory cells. J. Immunol. 168:365-371.

117.Fischer, K., et al. 2004. Mycobacterial phosphatidylinositol mannoside is a natural antigen for CD1d-restricted T cells. Proc. Natl. Acad. Sci.U. S. A. 101:10685-10690.

118.Korsgren, M., et al. 1999. Natural killer cells determine development of allergen-induced eosinophilic airway inflammation in mice. J. Exp. Med. 189:553-562.

119.Lisbonne, M., et al. 2003. Cutting edge: invariant
Valpha14 NKT cells are required for allergeninduced airway inflammation and hyperreactivity in an experimental asthma model. J. Immunol. 171:1637-1641.

120.Akbari, O., et al. 2003. Essential role of NKT cells producing IL-4 and IL-13 in the development of allergen-induced airway hyperreactivity. Nat. Med. 9:582-588.

121.Bilenki, L., Yang, J., Fan, Y., Wang, S., and Yang, X. 2004. Natural killer T cells contribute to airway eosinophilic inflammation induced by ragweed through enhanced IL-4 and eotaxin production. Eur. J. Immunol. 34:345-354.

122.Prell, C., et al. 2003. Frequency of Valpha24+CD161+ natural killer T cells and invariant TCRAV24-AJ18 transcripts in atopic and non-atopic individuals. Immunobiology. 208:367-380.

123.Campos, R.A., et al. 2003. Cutaneous immunization rapidly activates liver invariant Valpha14 NKT cells stimulating B-1 B cells to initiate T cell recruitment for elicitation of contact sensitivity. J. Exp. Med. 198:1785-1796.

124.Tupin, E., et al. 2004. CD1d-dependent activation of NKT cells aggravates atherosclerosis. J. Exp. Med. 199:417-422. 